\title{
RITMO DE CRECIMIENTO, PRODUCCIÓN DE BIOMASA Y DINÁMICA DE MOVILIZACIÓN DE CARBOHIDRATOS EN UN CULTIVO DE Adesmia bicolor (POIR.) DC (FABACEAE)
}

\author{
GROWTH RATE, BIOMASS PRODUCTION AND DYNAMICS OF \\ CARBOHYDRATE MOBILIZATION IN CULTIVATED Adesmia bicolor (POIR.) \\ DC (FABACEAE)
}

Verónica Pérez ${ }^{1}$, Mercedes Panzitta ${ }^{1}$, Carla Vidal ${ }^{1}$, Sara Basconsuelo ${ }^{1,2}$, Juan Gorjon ${ }^{1}$, Damaris Fitzimons ${ }^{1}$, Rosana Malpassi ${ }^{1,2}$, Luciana Bianco ${ }^{1,2 * *}$

\footnotetext{
${ }^{1}$ Laboratorio de Morfología Vegetal, Facultad de Agronomía y Veterinaria, Universidad Nacional de Río Cuarto, Ruta 36 km 601, (5800) Río Cuarto, Provincia de Córdoba, Argentina.

2 Instituto de Investigaciones Agrobiotecnológicas (INIAB CONICET-UNRC), Ruta 36 km 601, (5800) Río Cuarto, Provincia de Córdoba, Argentina.

*Autor para correspondencia E-mail: lbianco@ayv.unrc.edu.ar
}

\section{RESUMEN}

Las leguminosas perennes nativas son importantes en los pastizales de diversas partes del mundo y pueden contribuir a mejorar el valor nutritivo de los sistemas de pastoreo naturales y cultivados. En la región árida y semiárida central de Argentina se ha comenzado la domesticación de especies con potencial forrajero, como es el caso de Adesmia bicolor (Poir.) DC. El objetivo de este trabajo es evaluar un cultivo de Adesmia bicolor (Poir.) DC y relacionar el ritmo de crecimiento de la especie con la producción de biomasa y la dinámica de movilización de carbohidratos durante un ciclo de cultivo. Para el estudio del ritmo de crecimiento se seleccionaron plántulas y plantas adultas, en las que se registraron variables vegetativas y reproductivas cada 7 a 10 días. Cada 60 días se registró la biomasa aérea y subterránea en un área de $0,0625 \mathrm{~m}^{2}$ y la concentración de carbohidratos en órganos subterráneos. La baja relación tallo/raíz de la plántula y el crecimiento clonal de la planta adulta de $A$. bicolor favorecen el establecimiento y desarrollo del cultivo con un máximo crecimiento vegetativo en invierno-primavera, el cual está determinado por la ramificación de los ejes cotiledonares y profilares y el aumento de la longitud de sus entrenudos. El ciclo de vida indefinido garantiza una tasa fotosintética importante que permite no sólo el mantenimiento del cultivo, sino también suficiente concentración de carbohidratos en raíces y estolones para permitir su rápida recuperación durante el rebrote. Estos resultados indican que A. bicolor es una especie que presenta atributos deseables para la producción forrajera.

Palabras claves: leguminosas nativas, forrajeras, Adesmia bicolor, movilización de reservas.

\section{ABSTRACT}

Native perennial legumes are an important component of rangelands in many parts of the world and may contribute to improve the nutritional value of natural and cultivated grazing systems. In the arid and semi-arid central region of Argentina, the domestication of species with forage potential, such as Adesmia bicolor (Poir.) DC, has begun. The objective of this work was to evaluate a crop of Adesmia bicolor (Poir.) DC and relate crop growth to biomass production, and carbohydrate mobilization dynamics during one year. To evaluate growth dynamics, seedlings and adult plants

\footnotetext{
Recibido: 04 noviembre 2019. Aceptado: 05 febrero 2020.
} 
were selected, and vegetative and reproductive variables were recorded every 7 to 10 days. In addition, aerial and ground biomass samples were taken in an area of $0,0625 \mathrm{~m}^{2}$ every 60 days and processed to determine carbohydrate mobilization dynamics. The low stem:root ratio observed in the seedlings and the clonal growth of $A$. bicolor adults plants favor the establishment and development of the crop, reaching its maximum vegetative growth in winter/spring. This maximum growth is determined by high cotyledonary and prophyllar branching with increased internode length. The indefinite life cycle guarantees an important photosynthetic rate that allows for not only crop persistence, but also enough carbohydrate concentration in roots and stolons, which permits a rapid recovery during regrowth. These results indicate that $A$. bicolor is a species that has desirable attributes for forage production.

Key words: native legumes, forage, Adesmia bicolor, reserve mobilization.

\section{INTRODUCCIÓN}

El estudio de leguminosas nativas ha cobrado interés en los últimos años como alternativa económica para la restauración de pastizales, la producción de forraje en cultivos mixtos, como así también para mejorar la calidad de las pasturas en suelos de baja fertilidad (Bécquer et al., 2017). En algunas regiones, estas especies han sido eliminadas o reducidas radicalmente por el sobrepastoreo, amplio uso de herbicidas para el control de malezas, transición de vegetación abierta a comunidades de plantas herbáceas densas, o incluso arbolado por intervención antrópica (Whitbread et al., 2009), por lo que se ha incrementado el interés por recuperarlas.

En la región árida-semiárida central de Argentina se ha observado la escasez de especies forrajeras que se adapten a los inviernos secos, ventosos, fríos, y a suelos con bajo contenido de N (nitrógeno). Para dar respuesta a esta situación se han realizado diversos estudios que forman parte de la evaluación de especies leguminosas nativas con potencial forrajero con el objetivo de domesticarlas. Entre ellas se encuentra Adesmia bicolor (Poir.) DC, la cual se distribuye en Argentina, sur de Brasil, centro de Chile y Uruguay. En Argentina crece en provincias como Misiones, Corrientes, Entre Ríos, Santa Fe, Tucumán, Río Negro, Córdoba y San Luis, específicamente en ambientes serranos y pedemontanos (Basconsuelo et al., 2013).

Entre los estudios realizados en $A$. bicolor se encuentran observaciones de la morfología de la planta adulta en su ambiente natural, modo de reproducción, poder germinativo y viabilidad de sus semillas, determinación de parámetros básicos de calidad forrajera, y fijación biológica de N (Bianco et al., 2013; Vidal et al., 2016; Panzitta et al., 2018). Adesmia bicolor es herbácea, perenne, de ciclo indefinido (preferentemente invernal) y tolerante a bajos niveles de fósforo $(\mathrm{P})$ en el suelo (11 ppm) (Dodd y Orr, 1995; Pereira, 2011). Tiene eficiencia de fijación biológica de $\mathrm{N}$ del $59 \%$, y calidad nutricional (alta degradabilidad:
$53,5 \%$ a las 6 hs, alcanzando $84 \%$ a las 24 hs; bajo contenido de fibras: $25,5 \%$ fibra detergente neutro y 20,5\% fibra detergente ácido; bajo contenido de lignina detergente ácido: 6,24\%; y alto contenido de proteína bruta: $18,5 \%$ ) indican que presenta alto potencial para uso forrajero (Basconsuelo et al., 2013; Bianco et al., 2013; Vileta et al., 2013).

En la evaluación de una especie como cultivo, el desarrollo fenológico adquiere relevancia ya que involucra atributos importantes a tener en cuenta, entre ellos, la caracterización del tipo de plántula como punto de partida para el establecimiento del cultivo, y las variantes de ramificación, como son el desarrollo del eje primario y la dirección de crecimiento de los vástagos ligados directamente a la producción de biomasa (Acosta, 2012; Ríos, 2015). Otra característica importante a tener en cuenta es el desarrollo del sistema radical, ya que está estrechamente relacionado con la distribución de los recursos disponibles en los micrositios de suelo (Bengough et al., 2011).

En las plantas clonales, como A. bicolor, el rebrote luego del pastoreo depende de las pequeñas hojas jóvenes y los estolones, considerados estos últimos como órganos vegetativos importantes de almacenamiento de reservas orgánicas. En cultivos forrajeros, debido a la utilización de las hojas como órganos de cosecha, la acumulación de carbohidratos en los órganos de reserva se convierte en una prioridad para garantizar su actividad como fuentes en la removilización después de la defoliación o en la salida del período de latencia (Pagliaricci et al., 2002).

Conjuntamente con las características morfológicas señaladas anteriormente, el rendimiento de las leguminosas forrajeras perennes depende del manejo del cultivo (fecha de siembra, frecuencia de corte, fertilización nitrogenada, entre otros) y de las condiciones ambientales (disponibilidad de $\mathrm{N}$ en el suelo, déficit hídrico, fotoperiodo y temperatura) (Lüscher et al., 2014). Estas variables inducen grandes cambios en la adquisición de recursos y modifican factores endógenos de la planta, como 
los niveles de reservas orgánicas de la raíz o el número de meristemas activos, ambos indicadores de hibernación, rebrote, rendimiento y potencial de persistencia de las plantas (Bedoussac et al., 2015). Por este motivo, la caracterización de estas variables permite un mejor entendimiento de los mecanismos químicos y fisiológicos del metabolismo del almidón, el cual es considerado la reserva orgánica más importante en los vegetales (Martínez-Vilalta et al., 2016). La producción de biomasa total producida por un cultivo es el resultado de la acumulación neta de $\mathrm{CO}_{2}$ durante la estación de crecimiento y la asimilación de $\mathrm{CO}_{2}$ está influenciada por factores ambientales y genéticos. Los primeros involucran longitud de horas luz, temperatura, concentración de $\mathrm{CO}_{2}$ y $\mathrm{O}_{2}$, disponibilidad de agua y nutrientes (Andrae, 2012).

Scheffer-Basso et al. (2002) estudiaron dos especies de Adesmia estoloníferas: A. latifolia y $A$. punctacta. Ellos afirman que el hábito de crecimiento está relacionado a la producción de biomasa y a la persistencia en condiciones de campo. En especies estoloníferas, el patrón de acumulación y distribución de fotosintatos se caracteriza principalmente por la producción de hojas, mientras que la persistencia depende de la formación continua de estolones.

En $A$. bicolor si bien en los últimos años se han realizado estudios de caracterización morfológica y producción de biomasa en cultivo (Basconsuelo et al., 2013), para continuar con la evaluación de esta especie como forrajera se propuso asociar el ritmo de crecimiento de la especie con la dinámica de producción de biomasa y la de movilización de carbohidratos durante un ciclo de cultivo.

\section{MATERIALES Y MÉTODOS}

El ensayo se llevó a cabo en el campo experimental de la Facultad de Agronomía y Veterinaria de la Universidad Nacional de Río Cuarto, Río Cuarto, Córdoba, Argentina (33ํํํㅜ S; 64º17' O), desde abril de 2012 hasta abril de 2013. Se utilizó una parcela de $4 \times 15 \mathrm{~m}$ con el cultivo de A. bicolor (Poir.) DC (Fabaceae) de secano implantado en el año 2008. Debido a que A. bicolor es una especie estolonífera, la implantación se realizó a partir de porciones de tepes (champas) de esta especie de 0,50 × 0,50 × 0,10 m de profundidad, procedentes de la localidad de Villa Rumipal (provincia de Córdoba, Argentina, 32ำ1' S, 64²8' O). El diseño utilizado fue completamente al azar con tres repeticiones.

Para el estudio morfológico, a partir de la primera semana de septiembre de 2012, se identificaron en la parcela las plántulas originadas de la resiembra natural del cultivo, teniendo en cuenta la presencia de los dos cotiledones al ras del suelo como criterio para seleccionar las que se encontraban en una misma etapa fenológica. Del total de plántulas observadas en este estadio se seleccionaron tres para su seguimiento fenológico no destructivo, mientras que el resto fue utilizado, en algunos casos, para los estudios morfológicos destructivos. El período de muestreo se extendió desde la primera semana de septiembre de 2012 hasta febrero de 2013. En la primera parte del estudio (desde inicio de septiembre hasta la segunda semana de octubre) las observaciones se realizaron cada 7 a 10 días y los muestreos fueron destructivos para la observación del inicio de desarrollo del sistema radical; en el resto de los meses se tomaron los datos de la parte aérea cada 20 a 35 días en forma no destructiva. Al finalizar la etapa de desarrollo vegetativo (diciembre de 2012) se realizó un muestreo destructivo para caracterizar el sistema radical de la planta adulta. En cada fecha de muestreo se analizaron tres individuos, en ellos se registraron las siguientes variables en la parte caulinar: sistema de ramificación, grado de ramificación, ubicación de yemas de renuevo, ubicación y orientación de ramas aéreas y subterráneas; en la parte radical: grado de ramificación del sistema radical, longitud, diámetro y orientación de la raíz principal a inicios del desarrollo y en planta adulta. De los tres valores obtenidos para cada variable en cada fecha de muestreo se calculó un promedio, que fue utilizado para la descripción de la evolución fenológica de A. bicolor en cultivo.

Para evaluar la producción de biomasa de A. bicolor se tomaron en la parcela del ensayo tres muestras al azar de órganos aéreos (hojas y tallos) y subterráneos (raíces y estolones) en áreas de $0,0625 \mathrm{~m}^{2}$ cada una, cada 60 días durante un año. Éstas fueron llevadas a estufa a $60^{\circ} \mathrm{C}$ y, posteriormente, pesadas en balanza analítica hasta obtener peso constante. De los tres valores obtenidos de materia seca (aérea y subterránea) en cada fecha de muestreo se calculó un promedio que luego fue utilizado para la elaboración de la curva de producción de biomasa del cultivo.

Para cuantificar la movilización de carbohidratos totales se tomaron tres muestras al azar de 0,2 g de raíces y estolones cada 30 días. Las mismas fueron homogeneizadas en un mortero con nitrógeno $(\mathrm{N})$ líquido y se les agregó $1,5 \mathrm{~mL}$ de ácido tricloroacético al $1 \%$. Luego fueron trasvasadas a un tubo Falcon y centrifugadas durante $5 \mathrm{~min}$ a $750 \mathrm{rpm}$. Se tomó el sobrenadante en cada una de ellas y se centrifugó nuevamente. Posteriormente, se colocó una alícuota de $20 \mu \mathrm{L}$ de muestra en un tubo de Khan y se enrasó a un volumen final de $500 \mu \mathrm{L}$ con agua destilada. Se le agregó $500 \mu \mathrm{L}$ de 
solución acuosa de fenol al 5\% y 2,5 mL de ácido sulfúrico puro, con agitación en vórtex entre cada paso. Los tubos fueron sumergidos en un baño de agua hirviendo por $10 \mathrm{~min}$, luego se dejaron enfriar a temperatura ambiente. Finalmente, se realizó la lectura en espectrofotómetro a 488 $\mathrm{nm}$, la cual fue comparada con la curva patrón que se realizó previamente, como control, para la que se utilizaron diluciones de una solución de glucosa $\left(1 \mathrm{mg} \mathrm{mL}^{-1}\right)$ y como blanco $500 \mu \mathrm{L}$ de agua destilada (Lopez-Legarda et al., 2017). De los tres valores de concentración de carbohidratos totales obtenidos para cada mes de muestreo se calculó un promedio que fue utilizado para la elaboración de la curva de movilización de este compuesto.

\section{RESULTADOS Y DISCUSIÓN}

Ritmo de crecimiento de $A$. bicolor durante el primer año de cultivo

El primer estadio fenológico correspondió al desarrollo de la plántula de germinación epígea con dos cotiledones elípticos ubicados al ras del suelo, en el mismo la relación tallo/raíz fue de
1:3. El siguiente estadio considerado se desarrolló a los 14 días posteriores con la aparición del primer nomofilo en el eje principal, el cual estaba formado por tres pares de folíolos (medias de 0,2 $\mathrm{cm}$ de largo y $0,1 \mathrm{~cm}$ de ancho) conjuntamente con la aparición de las primeras raíces laterales a partir de la raíz principal, formando el sistema radical alorrizo (Fig. 1A).

Las plántulas son la base para interpretar las formas de crecimiento y a su vez se consideran una etapa crítica en el ciclo de vida de una planta. La combinación de factores bióticos y abióticos (densidad de vegetación, competencia intra e interespecífica, cobertura de suelo, humedad, temperatura e intensidad de luz) determina la posibilidad de que las semillas germinen y las plántulas se desarrollen (Veneciano et al., 2005). Vogel (1980) planteó que el establecimiento de una planta joven ocurre principalmente por la elongación de la raíz principal, desarrollo de raíces laterales y pelos radicales. En A. bicolor, si bien el tipo de plántula faneroepígea se considera vulnerable a factores externos, la alta relación raíz:parte aérea de la plántula permite un rápido establecimiento. Esta característica es
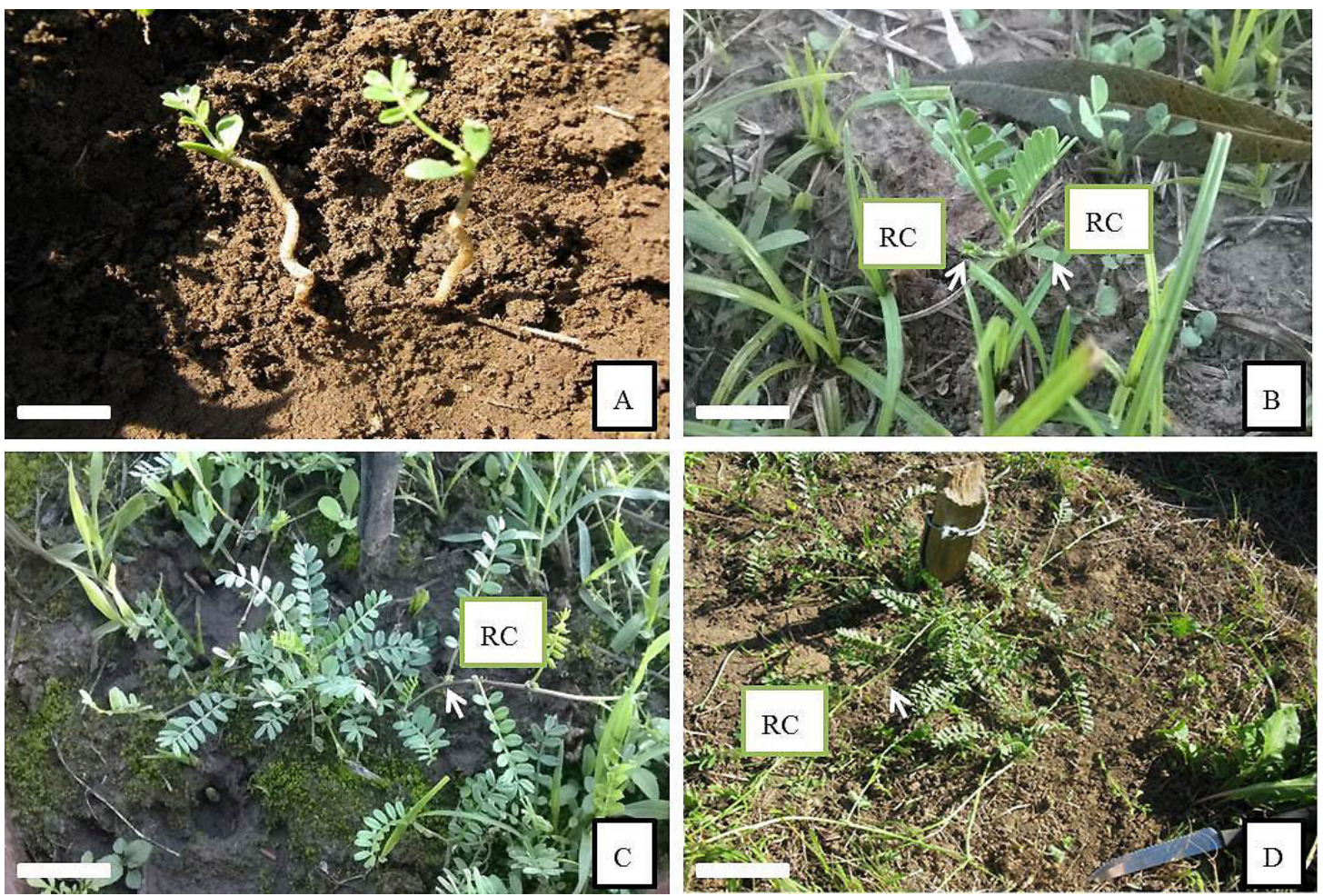

Fig. 1. Ejemplares de Adesmia bicolor en diferentes estadios. A. 14 días; B. 37 días; C. 60 días; D. 112 días desde el primer estadío. Año: 2012. Campo Experimental de la Facultad de Agronomía y Veterinaria, UNRC. RC: rama cotiledonar.

Fig. 1. Adesmia bicolor at different development stages. A: 14 days; B: 37 days; C: 60 days; D: 112 days from the first stage. Year: 2012. Experimental Station of the College of Agronomy and Veterinary Medicine, UNRC. RC: Cotyledon shoot. 
importante como se ha señalado en otros estudios de Leguminosas nativas, ya que está relacionada directamente con una buena implantación del cultivo (Vignolio y Fernández, 2011).

A los 28 días desde el primer estadio, se observaron tres nomofilos con cuatro pares de folíolos y una longitud media del pecíolo de 0,2 $\mathrm{cm}$. La raíz principal alcanzó una media de $5 \mathrm{~cm}$ de longitud y las laterales de $1 \mathrm{~cm}$. A los 37 días, se agregó un nomofilo y comenzaron a desarrollarse las dos ramas cotiledonares plagiótropas de 1,5 $\mathrm{cm}$ de longitud media (Fig. 1B), las raíces laterales alcanzaron $1,5 \mathrm{~cm}$ mientras que la principal mantuvo una media de $5 \mathrm{~cm}$. Luego de 45 días, las dos ramas cotiledonares plagiótropas alcanzaron entre 3 y $4 \mathrm{~cm}$ de longitud y en cada nudo se desarrolló una hoja compuesta de hasta 7 pares de folíolos con una yema vegetativa en su axila. El eje principal alcanzó una media de $1 \mathrm{~cm}$ de altura y los cotiledones aún persistían. La longitud media de la raíz principal aumentó a $5,5 \mathrm{~cm}$ y las laterales una media de $2,5 \mathrm{~cm}$. El siguiente estadio fue caracterizado a los 60 días a partir del primer estadio, cuando se produjo la abscisión de los cotiledones, el eje principal alcanzó una media de $4 \mathrm{~cm}$ de altura y las ramas cotiledonares medían $10 \mathrm{~cm}$ de longitud. Estas últimas fueron transformándose en estolones, en cada nudo se desarrollaron raíces adventicias comenzando por aquellos más cercanos al eje principal (Fig. 1C).

En plantas de 80 días se observó que el crecimiento de las ramas cotiledonares $(22 \mathrm{~cm}$ de longitud) fue más rápido que el del eje principal (4 a $5 \mathrm{~cm}$ de altura). En este estadio, la raíz principal alcanzó una media de $6 \mathrm{~cm}$ de longitud y las raíces laterales de mayor crecimiento de 4 $\mathrm{cm}$ de longitud.

En el siguiente estadio (112 días a partir del primer estadio), el eje principal midió entre 8 y $10 \mathrm{~cm}$ de altura, mientras que en las ramas cotiledonares de $30 \mathrm{~cm}$ de longitud aproximadamente comenzaron a desarrollarse las ramas profilares ( $2 \mathrm{~cm}$ de longitud media). En este periodo, la relación altura de tallo:longitud de raíz fue de 1:1 (Fig. 1D) y el diámetro de la raíz principal alcanzó una media de $0,2 \mathrm{~cm}$.

En plantas de 145 días desde el primer estadio, el eje principal mantuvo la altura de la etapa anterior, las ramas cotiledonares midieron 60 $\mathrm{cm}$ de longitud media y en sus últimos nudos se desarrollaron los primeros racimos a partir de yemas reproductivas (Fig. 2), marcando el inicio de la etapa reproductiva que culminó con la fructificación desde la última semana de febrero

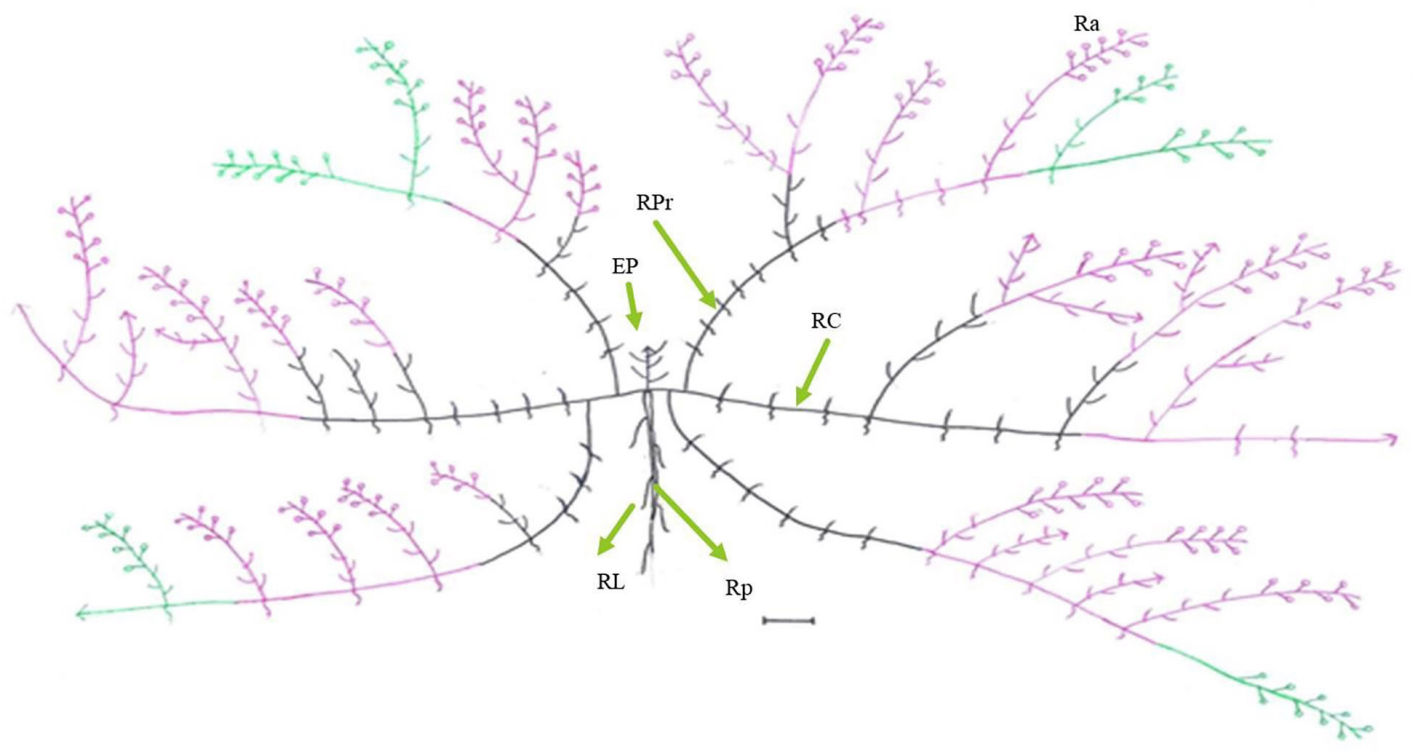

Fig. 2. Ritmo de crecimiento de A. bicolor en condiciones de campo durante el primer año de vida. Año: 2012. Campo Experimental de la Facultad de Agronomía y Veterinaria, UNRC.

Fig. 2. Growth rate of Adesmia bicolor under field conditions during the first growing cycle. Year: 2012. Experimental Station of the College of Agronomy and Veterinary Medicine, UNRC.

Línea negra: crecimiento en primavera; Línea rosa: crecimiento en verano; Línea Verde: crecimiento fines de veranoinicios de otoño; RA: racimo; RC: rama cotiledonar; RPR: rama profilar; EP: eje primario; RP: raíz principal; RL: raíz lateral. Escala: $4 \mathrm{~cm}$.

Black line: growth in spring; Pink line: growth in summer; Green Line: growth in late summer/early fall; RA: cluster; RC: cotyledon shoot; RPR: prophil shoot; EP: primary shoot; RP: main root; RL: lateral root. Scale: $4 \mathrm{~cm}$. 
y marzo. Los racimos presentaron un raquis de 8 $\mathrm{cm}$ de longitud media, con 8 a 10 flores sostenidas por pedicelos 0,5 a $1,0 \mathrm{~cm}$ de longitud. En este período, las ramas profilares alcanzaron una media de $25 \mathrm{~cm}$ de longitud y presentaron un nomofilo en cada nudo con una yema vegetativa.

Tanto las yemas vegetativas de las ramas cotiledonares como las de las profilares permanecieron al ras del suelo hasta retomar el período de crecimiento vegetativo, comportándose como una especie hemicriptófita. Al finalizar el otoño y durante el invierno, conjuntamente con el desarrollo de estas yemas en nuevas ramas, se produce un alargamiento de los entrenudos (1,2 a 1,9 cm), crecimiento en altura de los brotes y mayor ancho de los folíolos. Las ramas cotiledonares alcanzaron una longitud media de $100 \mathrm{~cm}$, mientras que las profilares 50 $\mathrm{cm}$.

Lüscher et al. (2014) destacaron la importancia de definir los módulos responsables del mantenimiento de las yemas de renuevo $y$, además, las características morfológicas y la respuesta de las yemas en función a las variaciones del ambiente. Teniendo en cuenta la importancia de estas características, se puede determinar que las subunidades arquitecturales (estolones) de A. bicolor se dispersan en función de la disponibilidad de recursos en los micrositios del suelo, y aprovechan el periodo favorable para colonizar áreas de mayor extensión. Además, el desarrollo de las raíces adventicias en los estolones favorece la adquisición de esos recursos. Ésto confirma lo expresado por diversos autores, quienes consideran que la morfología del sistema radical es un producto de la interacción entre genotipo-ambiente y resulta en una distribución de raíces en el espacio en función de los recursos (Bengough et al., 2011; Acosta, 2012; Río Valverde, 2015).

El marcado desarrollo y ramificación de las ramas plagiótropas en relación al eje principal observado en este trabajo, confirma lo expresado por Veneciano et al. (2005) quienes caracterizaron a A. bicolor como especie colonizadora. Además, su estructura estolonífera no sólo favorece el aumento de la eficiencia fotosintética y la distribución de nutrientes, agua y fotoasimilados (Basconsuelo et al., 2013), sino que constituye un mecanismo de protección de las yemas de renuevo frente a pastoreo intensivo. Todas estas características favorecerían la persistencia del cultivo.

La Fig. 2 muestra el ritmo de crecimiento de $A$. bicolor obtenido a partir de la secuenciación de las distintas etapas de desarrollo. Se observa que los períodos de invierno y primavera son las etapas de máximo crecimiento vegetativo, y verano- comienzos de otoño corresponde al período reproductivo. A diferencia de lo que ocurre en A. latifolia y A. punctacta (ambas estoloníferas), en las que el aumento en la producción de biomasa aérea coincide con un mayor desarrollo de hojas (Scheffer- Basso et al., 2002), en A. bicolor el incremento en la producción de biomasa aérea ocurre además por la ramificación de los ejes cotiledonares y profilares, y el aumento de la longitud de sus entrenudos.

\section{Producción de biomasa y dinámica de movilización de carbohidratos en $A$. bicolor}

La Fig. 3 muestra la producción de biomasa a lo largo del ciclo del cultivo de A. bicolor, en la que se puede observar que en mayo y en octubre se produjeron los mayores valores medios de producción de biomasa aérea $(881,6$ y $891,2 \mathrm{~kg} \mathrm{ha}^{-1}$, respectivamente). Entre estos picos de máxima producción, se observó que en julio disminuyó a la mitad (441,6 $\left.\mathrm{kg} \mathrm{ha}^{-1}\right)$. Teniendo en cuenta los registros de temperatura y precipitaciones del ambiente donde se realizó la experiencia, esta disminución coincidió con un período en el que las precipitaciones fueron escasas (Tabla 1). A partir de octubre comienza a disminuir progresivamente hasta llegar a abril, momento en que se observó la menor producción de biomasa aérea (208 $\left.\mathrm{kg} \mathrm{ha}^{-1}\right)$.

Por otro lado, la biomasa subterránea media producida a lo largo del ciclo del cultivo de $A$. bicolor (Fig. 3) muestra que los mayores niveles también ocurrieron en los meses de mayo y octubre (1339,2 y 1254,4 $\mathrm{kg} \mathrm{ha}^{-1}$, respectivamente), mientras que los menores se registraron en el mes de abril (340,8 $\left.\mathrm{kg} \mathrm{ha}^{-1}\right)$. Por lo tanto, los picos de producción de biomasa aérea y subterránea coinciden en el tiempo, ya que en mayo y octubre se observan los dos picos de mayor producción y en abril el de menor producción.

Estos resultados confirman lo establecido por Pereira (2011), quien califica a A. bicolor como una especie de ciclo indefinido preferentemente invernal. En este caso se observó que puede retrasar su máximo crecimiento vegetativo hasta períodos en los que la disponibilidad de humedad en el suelo sea mayor. Los valores obtenidos en mayo y octubre concuerdan con un aumento en las precipitaciones, lo que indicaría que la especie podría ser más afectada por la falta de agua en el sustrato que por las condiciones óptimas de temperatura. En relación a esto último se observa una diferencia respecto al patrón descripto para alfalfa, ya que en abril las plantas aumentan rápidamente su biomasa en raíces y estolones y luego, al mes siguiente, aumentan recién su biomasa aérea (Mendoza et al., 2010). Esto probablemente se deba a las diferentes 


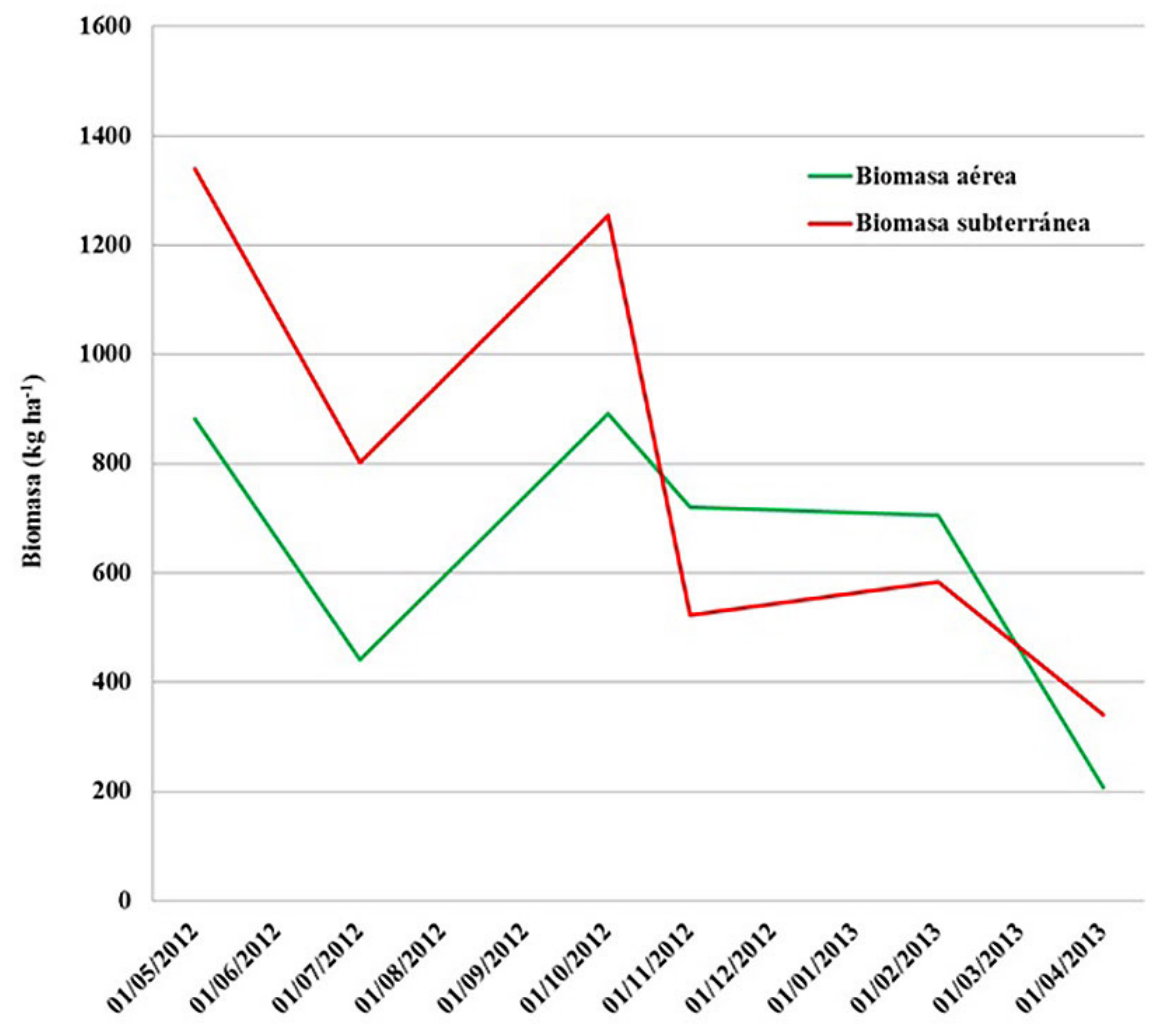

Fig. 3. Producción media de biomasa aérea y subterránea de $A$. bicolor durante un año de cultivo. Fig. 3. Mean aerial and underground biomass of cultivated A. bicolor in one year of production.

Tabla 1. Temperaturas media mensual y precipitaciones medias para el período Abril 2012-Mayo 2013. Datos registrados en la Estación del Campo Experimental de la Facultad de Agronomía y Veterinaria, Río Cuarto, Argentina.

Table 1. Average monthly temperatures and average rainfall for the period April 2012-May 2013. Data recorded at the Experimental Station of the Faculty of Agronomy and Veterinary Medicine, Rio Cuarto, Argentina.

\begin{tabular}{lccc}
\hline Mes & Año & $\begin{array}{c}\text { Temperatura media } \\
\left({ }^{\circ} \mathbf{C}\right)\end{array}$ & $\begin{array}{c}\text { Precipitaciones } \\
(\mathbf{m m})\end{array}$ \\
\hline Abril & 2012 & 16,6 & 78 \\
Mayo & 2012 & 14,4 & 17 \\
Junio & 2012 & 9,6 & 0 \\
Julio & 2012 & 8,0 & 1 \\
Agosto & 2012 & 11,4 & 13 \\
Septiembre & 2012 & 14,5 & 84 \\
Octubre & 2012 & 16,3 & 140 \\
Noviembre & 2012 & 20,6 & 166 \\
Diciembre & 2012 & 22,8 & 104 \\
Enero & 2013 & 23,4 & 76 \\
Febrero & 2013 & 219 & 64 \\
Marzo & 2013 & 18,1 & 101 \\
Abril & 2013 & 17,9 & 43 \\
Mayo & 2013 & 13,0 & 14 \\
\hline Gentileza Laboratorio de Agrometeorología (UNRC).
\end{tabular}

Gentileza Laboratorio de Agrometeorología (UNRC). 
estructuras de los órganos subterráneos que desarrollan estas especies.

La Fig. 4 muestra la concentración de carbohidratos totales en raíces y estolones de A. bicolor a lo largo del ciclo del cultivo (sin fertilización, riego o defoliación). En ella se puede observar que comenzó a aumentar en el mes de julio $\left(0,43 \mathrm{mg} \mathrm{g}^{-1}\right.$ de muestra) hasta alcanzar su mayor valor en el mes de octubre $(0,49 \mathrm{mg}$ $\mathrm{g}^{-1}$ de muestra) en coincidencia con la mayor producción de biomasa (aérea y subterránea). Luego la concentración de carbohidratos disminuyó progresivamente hasta alcanzar el valor mínimo en febrero $\left(0,30 \mathrm{mg} \mathrm{g}^{-1}\right.$ de muestra). A partir de este momento aumentó nuevamente hasta mayo $\left(0,43 \mathrm{mg} \mathrm{g}^{-1}\right.$ de muestra).

$\mathrm{Al}$ asociar todas las variables estudiadas en A. bicolor, se observa que los máximos valores de reserva de carbohidratos en raíces y estolones registrados en mayo y entre agosto y noviembre coincidieron con la mayor producción de biomasa aérea. Asimismo, la disminución progresiva a partir de noviembre indicaría el comienzo de la etapa reproductiva con una alta demanda de fotoasimilados. Esta situación se revierte a partir de marzo (final del período reproductivo), momento en el que comienza un rápido incremento de concentración de carbohidratos que marcarían la recuperación de las reservas del cultivo. Esto se debería, por un lado, a la presencia de estolones que facilitan la movilidad de hidratos de carbono en la planta $\mathrm{y}$, por otro, a la perennidad del follaje. Esta última característica determina que la tasa fotosintética nunca sea nula, a pesar de que haya períodos en los que disminuye y afecte la producción de reservas. Por lo tanto, el período de disminución del crecimiento es menos severo que lo planteado para especies perennes en las que muere totalmente la parte aérea.

\section{CONCLUSIONES}

El ritmo de crecimiento de $A$. bicolor corresponde a una especie de ciclo de vida indefinido, con un período de alto crecimiento y desarrollo de órganos aéreos y subterráneos que permite no sólo el mantenimiento del cultivo, sino la concentración de carbohidratos en raíces y estolones.

Estos atributos sumados a las características morfológicas que garantizan el establecimiento y la persistencia del cultivo determinan que $A$. bicolor es una especie con caracteres deseables para la producción forrajera.

\section{LITERATURA CITADA}

Acosta, J.M. 2012. Arquitectura y modelos de crecimiento de malezas tolerantes a glifosato. Caracterización de las posibles estrategias de escape a la acción herbicida. Tesis Doctorado en Ciencias Biológicas. Facultad de Ciencias Agrarias-Instituto de Agrobiotecnología del Litoral (UNL-CONICET), Santa Fe, Argentina.

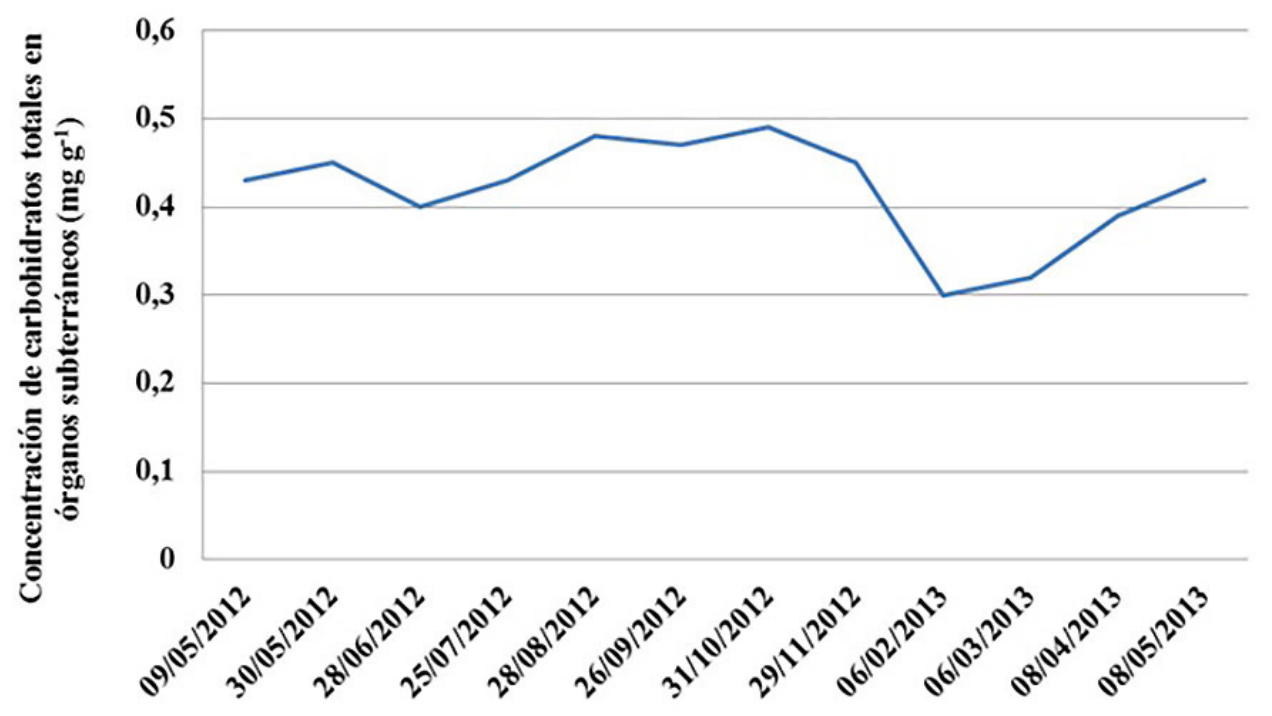

Fig. 4. Concentración media de carbohidratos totales en raíces y estolones de A. bicolor a lo largo del ciclo del cultivo.

Fig. 4. Mean concentration of total carbohydrates in roots and stolons of $A$. bicolor over a growing cycle. 
Andrae, J. 2012. Grazing impacts on pasture composition. UGA Cooperative Extension Bulletin 1243. 4 p. University of Georgia, Georgia, USA.

Basconsuelo, S., M. Grosso, T. Kraus, C. Bianco, L. Bianco, y D. Vileta. 2013. Leguminosas nativas con potencial forrajero: Adesmia bicolor. E-book. UniRío, Río Cuarto, Argentina. Disponible en https://www. unrc.edu.ar/unrc/comunicacion/editorial/ repositorio/978-987-688-038-1.pdf(Verificado 24 octubre 2019)

Bécquer, C.J., Y. Galdol, A. Mirabal, M. Quintana, and A. Puentes. 2017. Rhizobia isolated from forage legumes of an arid cattle rearing ecosystem in Holguín, Cuba. Tolerance to abiotic stress and catalase production (Phase II). Cuban Journal Agr. Sci. 51:117-127.

Bedoussac, L., E. Pascal Journet, H. HauggaardNielsen, C. Naudin, G. Corre-Hellou, E. Steen Jensen, et al. 2015. Ecological principles underlying the increase of productivity achieved by cereal-grain legume intercrops in organic farming. A review. Agron. Sustain. Dev. 35:911-935.

Bengough, A.G., B.M. McKenzie, P.D. Hallett, and T.A. Valentine. 2011. Root elongation, water stress, and mechanical impedance: a review of limiting stresses and beneficial root tip traits. J. Exp. Bot. 62:59-68.

Bianco, L., J. Angelini, A. Fabra, and R. Malpassi. 2013. Diversity and symbiotic effectiveness of indigenous rhizobia-nodulating Adesmia bicolor in soils of Central Argentina. Curr. Microbiol. 66:174-184.

Dodd, M.B., and S.J. Orr. 1995. Seasonal growth, phosphate response, and drought tolerance of 11 perennial legume species grown in a hill-country soil. New Zealand J. Agric. Res. 38:7-20.

López-Legarda, X., A. Taramuel-Gallardo, C. Arboleda-Echavarría, F. Segura-Sánchez, y L.F. Restrepo-Betancur. 2017. Comparación de métodos que utilizan ácido sulfúrico para la determinación de azúcares totales. Rev. Cubana Quím. 29:180-198.

Lüscher, A., I. Mueller-Harvey, J.F. Soussana, R.M. Rees, and J.L. Peyraud. 2014. Potential of legume-based grassland-livestock systems in Europe: a review. Grass Forage Sci. 69:206-228. doi: 10.1111/gfs.12124.

Martínez-Vilalta, J., A. Sala, D. Asensio, L. Galiano, G. Hoch, S. Palacio, et al. 2016. Dynamics of non-structural carbohydrates in terrestrial plants: a global synthesis. Ecol. Monogr. 86:495-516.
Mendoza P., S.I., A. Hernández Garaya, J. Pérez Péreza, A.R. Quero Carrilloa, J.A.S. Escalante Estrada, y J.L. Zaragoza Ramírez. 2010. Respuesta productiva de la alfalfa a diferentes frecuencias de corte. Rev. Mex. Cienc. Pecu. 1:287-296.

Pagliaricci, H.R., T. Pereyra, A. Ohanian, y S. González. 2002. Utilización de pasturas. 28 p. Sitio Argentino de Producción Animal. Disponible en https:// documentop.com/modulo-utilizacion-depasturas-sitio-argentino-de-produccionanimal_5a12d3511723dd246b1f4a5a.html

Panzitta, M. 2018. Determinación de la fijación biológica de nitrógeno de Adesmia bicolor (Leguminosae) en condiciones de campo. Tesis Ingeniería Agronómica. Facultad de Agronomía y Veterinaria, Universidad Nacional de Río Cuarto, Río Cuarto, Argentina.

Pereira M., M.P. 2011. Manejo y conservación de pasturas naturales de Basalto. Reedición. Programas de servicios agropecuarios MGAP-BID. Ministerio de Agricultura, Ganadería y Pesca-Programa Ganadero. Instituto Plan Agropecuario, Montevideo, Uruguay.

Ríos V., P.A. 2015. Hábitos de crecimiento y forma de copa en la fijación de dióxido de carbono equivalente $\left(\mathrm{CO}_{2} \mathrm{e}\right)$ en especies vegetales de un sistema multiestrato. Fundo UNAP. Distrito de San Juan Bautista. Loreto. 2015. Tesis Ingeniero en Gestión Ambiental. Facultad de Agronomía, Universidad Nacional de la Amazonía Peruana, Iquitos, Perú.

Scheffer-Basso, S.M., A.V. Avila Jacques, e Y.M. Dall'Agnol. 2002. Alocação da biomassa e correlações morfofisiológicas em leguminosas forrageiras com hábitos de crescimento contrastantes. Sci. Agric. 59:629634.

Veneciano, J.H., C.A. Frasinelli, T.A. Kraus, y C.A. Bianco. 2005. Domesticación de especies forrajeras. 64 p. Ed. Universidad Nacional de Río Cuarto, Córdoba, Argentina.

Vidal, C., M. Ibañez, G. Boito, C. Crenna, y S. Basconsuelo. 2016. Sistema reproductivo de Adesmia bicolor (Leguminosae). Interciencia 41:757-762.

Vignolio, O., y O. Fernández 2011. Lotus tenuis seedling establishment and biomass production in flooding pampa grasslands (Buenos Aires, Argentina). Chilean Journal of Agricultural Research 71(1):96-103. 
Vileta, D., M. Grosso, and M. Fondevila. 2013. Nutritive value for ruminants of two herbaceous South-American native legumes: Adesmia bicolor and A. macrostachya. Anim. Prod. Sci. 54:125-130.

Vogel, E. 1980. Seedlings of Dicotyledons. 276 p. Centre for Agricultural Publishing and Documentation, Wageningen, The Netherlands.

Whitbread, A.M., C.A. Hall, and B.C. Pengelly. 2009. A novel approach to planting grasslegume pastures in the mixed farming zone of southern inland Queensland, Australia. Crop Pasture Sci. 60:1147-1155. doi.org/10.1071/ CP09058 